\title{
Current clinical management of smoke inhalation injuries: a reality check
}

\author{
Arietta Spinou $\mathbb{1}^{1}$ and Nikolaos G. Koulouris (i) ${ }^{2}$ \\ Affiliations: ${ }^{1}$ Health Sport and Bioscience, University of East London, London, UK. ${ }^{2} 1$ st Respiratory Medicine \\ Dept, National and Kapodistrian University of Athens, Medical School, Athens, Greece.
}

Correspondence: Arietta Spinou, University of East London, Health Sport and Bioscience, Water Lane, Stratford, London E15 4LZ, UK. E-mail: a.spinouduel.ac.uk

@ERSpublications

Smoke inhalation injury is a complex clinical condition and respiratory clinicians need to have a good understanding of its current clinical management. However, evidence derives mostly from retrospective cohorts and case series. Is this enough? http://ow.ly/PrtT30mJcYD

Cite this article as: Spinou A, Koulouris NG. Current clinical management of smoke inhalation injuries: a reality check. Eur Respir J 2018; 52: 1802163 [https://doi.org/10.1183/13993003.02163-2018].

A major disaster is happening at the moment, as the Camp Fire, Woolsey Fire and Hill Fire are burning in California. Camp Fire in Northern California has already burned $546.3 \mathrm{~km}^{2}$ and is the deadliest wildfire in the history of the state, with 48 fatalities and still counting [1]. It was also only recently, in July 2018, when a fire entered the populated area of Mati, Greece, and created a wildland urban interface that caused 99 fatalities and numerous burns and smoke inhalation injuries. A few years ago, in August 2007, 67 people died in a megafire event in the Peloponnese region, Greece, which was created by 55 simultaneous large fires (based on size, intensity, environmental and socio-economic impact) [2]. These and numerous other tragic incidents highlight the importance of our current clinical management of the victims of fire.

Burns are categorised according to depth, and their severity depends on the extent (percentage of total body surface area), age of the individual and accompanied smoke inhalation. Essential treatment for burn victims remains resuscitation with intravenous fluids to maintain optimal fluid balance, nutrition optimisation, wound coverage and pain control [3]. Smoke inhalation injury increases mortality and is the most common cause of death in the event of fire [4]. This type of injury is defined as the "damage caused by breathing in harmful gases, vapours, and particulate matter contained in smoke" [4] and is categorised as: 1) thermal, usually involving damage of the upper respiratory tract by the high temperature of smoke and gases; 2) chemical, usually involving damage of the lower respiratory system from the inhalation of irritant fire by-products and particulate matter deposition; 3) asphyxiation and systemic toxicity, caused by inhalation of deleterious gases produced by combustion, usually carbon monoxide and hydrogen cyanide; or 4) a combination of the above [5].

Diagnosis of smoke inhalation injury is based on history, signs and symptoms, and fibreoptic bronchoscopy, although parenchyma damage in some cases can be present even with negative bronchoscopy findings [6]. Clinical indicators of smoke inhalation are: facial burns; lip, tongue, mouth, pharynx or nasal mucosa burns; singeing of nasal hair or eyebrows; oropharyngeal acute inflammatory changes; carbonaceous particles containing toxic chemicals (soot) in the oropharynx; and signs of airway obstruction, irritation or damage. A history of confusion, reduced level of consciousness or unconsciousness, and/or entrapment in a burning location, and carboxyhaemoglobin level $>10 \%$ are additional indicators. Symptoms can be present on hospital admission or develop up to $48 \mathrm{~h}$ post-burn, and they include dyspnoea, evidence of increased work of breathing, hoarseness, wheeze, stridor and 
ronchi, productive cough and soot stained/carbonaceous sputum [4]. Bronchoscopy visualises the severity of inhalation injury, permits grading through the Abbreviated Injury Score, and uses washout fluid for pulmonary hygiene, microscopy and culture [7]. Normal findings on chest radiography or oxygenation do not exclude injury [5].

Smoke inhalation injury can be critical and life-threatening, and there is a risk of rapid deterioration and admission to the intensive care unit [4]. Patients should be continuously monitored and assessed, particularly for upper airway obstruction, as oedema can present up to $36 \mathrm{~h}$ post-burn $[4,5]$. The length of exposure, generated temperatures and micro-environment of a fire, i.e. the scene, its materials, the availability of oxygen, and the nature of the combustion, dictate the composition of gases and severity of the injury [4]. Chemical irritants, along with toxic by-products, damage the airway epithelium and introduce an inflammatory response with neutrophil infiltration and fibrinogen activation, which can lead to oedema and bronchospasm. Dead epithelial cells, inflammatory cells, mucous and fibrin formulate airway casts. These casts cause various degrees of mechanical airway obstruction, increase resistance and reduce lung compliance, therefore increase the work of breathing and generate ventilation/perfusion $\left(V^{\prime} / Q^{\prime}\right)$ mismatch [8].

To ensure airway patency, particularly in the development of tongue and tracheal oedema and stenosis, patients may require intubation by endotracheal tube or tracheostomy and mechanical ventilation [9]. Ventilator-associated pneumonia is a common complication and people with extensive burns may present pulmonary oedema or acute respiratory distress syndrome (ARDS). Since positive pressure mechanical ventilation is associated with ventilator-associated lung injury and ARDS, clinicians can apply lung protective strategies when this is compatible with the respiratory demands of the patient [5]. Non-conventional ventilation strategies include the high-frequency percussive ventilation, prone positioning and extracorporeal membrane oxygenation, but evidence does not support their routine use [10-13]. Patients in respiratory failure, during the weaning process or self-ventilated individuals with reduced chest wall elasticity and lung compliance, may require noninvasive ventilation (NIV) [14]. In the application of the NIV, patient compliance and special contraindications to NIV, such as facial burns, should be considered [13].

Indeed, protecting gas exchange is paramount. Carbon monoxide from the fire scene usually causes hypoxaemia, which in closed environments worsens through consumption of oxygen by the fire. Additionally, patients with burns present hypermetabolic response and, therefore, high respiratory demands. Loss of the physiological hypoxic pulmonary vasoconstriction also results in $V^{\prime} / Q^{\prime}$ mismatch. Restoring hypoxaemia reduces the $V^{\prime} / Q^{\prime}$ mismatch and shifts the oxygen dissociation curve to the right, thus the increase of arterial oxygen partial pressure accelerates carbon monoxide displacement from haemoglobin at the tissue level [5]. High fraction of inspired oxygen is provided, initially $100 \%$ and then at the lowest acceptable level. The addition of humidification facilitates airway clearance, particularly in the presence of copious carbonaceous secretions [6]. Inhaled nitric oxide can be used to improve arterial oxygen partial pressure in acute respiratory failure, and exogenous surfactant to enhance the compliance of alveoli $[15,16]$. Furthermore, high flow oxygen serves as an "antidote" to inhaled toxic substances, such as to hydrogen cyanide poisoning. Hydroxocobalamin is the true antidote to hydrogen cyanide, but it must be administered immediately following the inhalation injury. For carbon monoxide poisoning, hyperbaric oxygen therapy has been suggested, but is not routinely recommended $[10,17]$.

Airway clearance is another important part of patient management [18]. Pain, drugs and artificial airways are common in patients with burns and can affect their breathing and cough effectiveness [19, 20]. Mucus, inactivation of surfactant, sedation and anaesthesia for surgical procedures, such as burn excision of the eschar and skin grafting, increase the risk of sputum retention, atelectasis and, consequently, pneumonia [6]. The epithelial damage triggers goblet cells to produce higher volume of secretions or foamy mucus, thus adversely affecting mucociliary clearance, and bronchial cast sloughing increases the risk of mucus plugging [21]. Use of techniques such as positioning or postural drainage, manual techniques, and manual or ventilator hyperinflation have been suggested as respiratory physiotherapy treatment options. Positioning can promote airway clearance and facilitate optimisation of $V^{\prime} / Q^{\prime}$ matching, and therefore improve oxygenation. Postural drainage can be part of positioning. This involves a group of gravity-assisted positions that promote mucus movement downwards (towards the mouth). Special contraindications for head-down positions are facial oedema and upper airway burns, which are usually nursed in the semi-upright position with a moderate elevation of the head and trunk [5]. Physiotherapists may apply manual techniques such as percussion, and any other manual handling, whilst avoiding shearing forces close to newly grafted or escharotomy areas. In chest wall burns, clinicians consider the burn scar depth, graft type, healing and viability of the tissue in the area, before performing their treatment. Moreover, manual or mechanical hyperinflation aims to promote airway clearance, maintain thoracic expansion and mobility, and prevent atelectasis [22]. In patients presenting weak and ineffective 


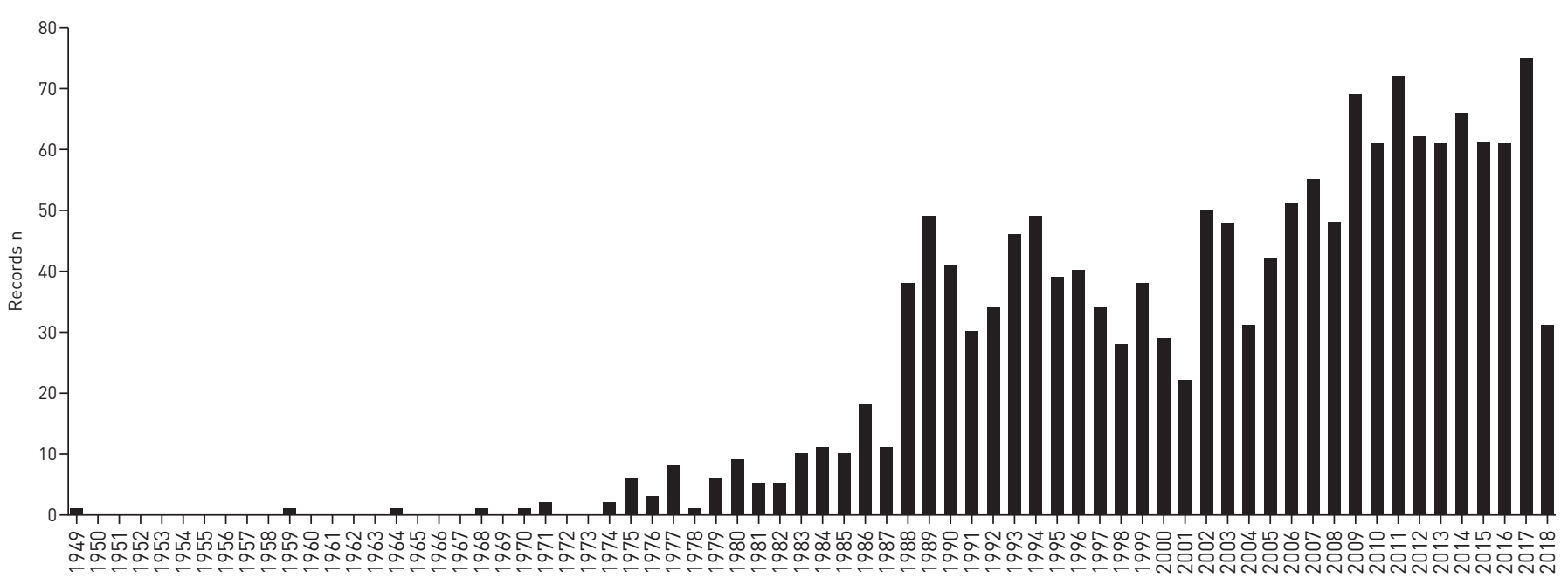

FIGURE 1 Number of PubMed findings per year, using the keywords "smoke inhalation injury". Data accessed October 2018.

cough or those who are unable to perform the manoeuvre, suction can accompany mucus mobilisation, using the nasopharyngeal, oropharyngeal, endotracheal tube or tracheostomy routes [23]. Airway clearance techniques are initiated after treatment for pain management, bronchodilators, and mucolitics such as $\mathrm{N}$ acetylcysteine, and before inhaled anticoagulants such as heparin, and antibiotic medications [24, 25]. Still, prophylactic antibiotics and corticosteroids are not indicated in this group of patients [5].

Overall, treatments aim to maximise effectiveness and avoid fatigue, and are tailored to the individual clinical presentation and potential underlying respiratory diagnosis and cardiovascular instability. Some patients can present full recovery from smoke inhalation injury without any remaining long term respiratory symptoms, although this depends on factors such as the smoke composition or the definition of smoke inhalation [26-28]. Mobilisation, early rehabilitation, activities of daily living and cardiorespiratory fitness training are important, and are prioritised at various stages of the clinical pathway [29]. Patients may also require psychological support $[30,31]$. Smoke inhalation injury is a complex clinical condition that needs careful management from a multidisciplinary team. Still, evidence for its clinical management is relatively limited, despite the increasing number of publications throughout the years (figure 1). We base our current practice mainly on retrospective cohorts and case series, rather than large scale clinical studies [13]. Is this level of evidence or the principles of physiology enough to guide most of our clinical decisions? Real life shows us that we urgently need to move towards more research and get stronger evidence for clinical application in the area.

Conflict of interest: None declared.

\section{References}

1 Cal Fire, State of California. Camp Fire Incident Information. 2018. www.fire.ca.gov/current_incidents/incidentdetails/ Index/2277 Date last accessed: November 14, 2018.

2 San-Miguel-Ayanz J, Moreno JM, Camia A. Analysis of large fires in European Mediterranean landscapes: lessons learned and perspectives. Forest Ecol Manag 2013; 294: 11-22.

3 Rowan MP, Cancio LC, Elster EA, et al. Burn wound healing and treatment: review and advancements. Crit Care 2015; 19: 243.

$4 \quad$ Gill P, Martin RV. Smoke inhalation injury. BJA Educ 2015; 15: 143-148.

ISBI Practice Guidelines Committee. ISBI practice guidelines for burn care. Burns 2016; 42: 953-1021.

Mlcak RP, Suman OE, Herndon DN. Respiratory management of inhalation injury. Burns 2007; 33: 2-13.

Endorf FW, Gamelli RL. Inhalation injury, pulmonary perturbations, and fluid resuscitation. J Burn Care Res 2007; 28: 80-83.

8 Enkhbaatar P, Pruitt BA, Suman O, et al. Pathophysiology, research challenges, and clinical management of smoke inhalation injury. Lancet 2016; 388: 1437-1446.

9 Aggarwal S, Smailes S, Dziewulski P. Tracheostomy in burns patients revisited. Burns 2009; 35: 962-966.

10 Walker PF, Buehner MF, Wood LA, et al. Diagnosis and management of inhalation injury: an updated review. Crit Care 2015; 19: 351.

11 Szentgyorgyi L, Shepherd C, Dunn KW, et al. Extracorporeal membrane oxygenation in severe respiratory failure resulting from burns and smoke inhalation injury. Burns 2018; 44: 1091-1099.

12 Miller AC, Ferrada PA, Kadri SS, et al. High-frequency ventilation modalities as salvage therapy for smoke inhalation-associated acute lung injury: a systematic review. J Intensive Care Med 2018; 33: 335-345. 
13 Deutsch CJ, Tan A, Smailes S, et al. The diagnosis and management of inhalation injury: an evidence based approach. Burns 2018; 44: 1040-1051.

14 Smailes ST. Noninvasive positive pressure ventilation in burns. Burns 2002; 28: 795-801.

15 Adhikari NKJ, Burns KEA, Friedrich JO, et al. Effect of nitric oxide on oxygenation and mortality in acute lung injury: systematic review and meta-analysis. BMJ 2007; 334: 779.

16 Pallua N, Warbanow K, Noah EM, et al. Intrabronchial surfactant application in cases of inhalation injury: first results from patients with severe burns and ARDS. Burns 1998; 24: 197-206.

17 Annane D, Chadda K, Gajdos P, et al. Hyperbaric oxygen therapy for acute domestic carbon monoxide poisoning: two randomized controlled trials. Intensive Care Med 2011; 37: 486-492.

18 Sheridan RL. Fire-related inhalation injury. N Engl J Med 2016; 375: 464-469.

19 Smailes ST, Martin RV, McVicar AJ. The incidence and outcome of extubation failure in burn intensive care patients. J Burn Care Res 2009; 30: 386-392.

20 Smailes ST, Martin RV, McVicar AJ. Evaluation of the spontaneous breathing trial in burn intensive care patients. Burns 2009; 35: 665-671.

21 Dries DJ, Endorf FW. Inhalation injury: epidemiology, pathology, treatment strategies. Scand J Trauma Resusc Emerg Med 2013; 21: 31.

22 Reper P, van Looy K. Chest physiotherapy using intrapulmonary percussive ventilation to treat persistent atelectasis in hypoxic patients after smoke inhalation. Burns 2013; 39: 192-193.

23 Smailes ST, McVicar AJ, Martin R. Cough strength, secretions and extubation outcome in burn patients who have passed a spontaneous breathing trial. Burns 2013; 39: 236-242.

24 Miller AC, Elamin EM, Suffredini AF. Inhaled anticoagulation regimens for the treatment of smoke inhalation-associated acute lung injury: a systematic review. Crit Care Med 2014; 42: 413-419.

25 Holt J, Saffle JR, Morris SE, et al. Use of inhaled heparin/N-acetylcystine in inhalation injury: does it help? J Burn Care Res 2008; 29: 192-195.

26 Bourbeau J, Lacasse Y, Rouleau MY, et al. Combined smoke inhalation and body surface burns injury does not necessarily imply long-term respiratory health consequences. Eur Respir J 1996; 9: 1470-1474.

27 Orozco-Levi M, Garcia-Aymerich J, Villar J, et al. Wood smoke exposure and risk of chronic obstructive pulmonary disease. Eur Respir J 2006; 27: 542-546.

28 Kurmi OP, Arya PH, Lam KB, et al. Lung cancer risk and solid fuel smoke exposure: a systematic review and meta-analysis. Eur Respir J 2012; 40: 1228-1237.

29 Hundeshagen G, Suman OE, Branski LK. Rehabilitation in the acute versus outpatient setting. Clin Plast Surg 2017; 44: 729-735.

30 Van Loey NE, Maas CJ, Faber AW, et al. Predictors of chronic posttraumatic stress symptoms following burn injury: results of a longitudinal study. J Trauma Stress 2003; 16: 361-369.

31 Dyster-Aas J, Willebrand M, Wikehult B, et al. Major depression and posttraumatic stress disorder symptoms following severe burn injury in relation to lifetime psychiatric morbidity. J Trauma 2008; 64: 1349-1356. 